\title{
CAN YOU SIMULATE TRAFFIC PSYCHOLOGY? AN ANALYSIS
}

\author{
Marco Lützenberger \\ Sahin Albayrak \\ Technische Universität Berlin, Distributed Artificial Intelligence Laboratory \\ Ernst-Reuter-Platz 7 \\ 10587 Berlin, GERMANY
}

\begin{abstract}
Contemporary traffic simulation frameworks use sophisticated physical- or mathematical models to "mimic" traffic systems in a lifelike fashion. Nevertheless, when it comes to road traffic, there seems to be no parameter more essential than the driver himself. Most frameworks neglect human factors in traffic entirely, or "estimate" a particular form of human behavior without providing any connection to reality. In this work we aim to establish such connection. We explain driver behavior from a psychological perspective and analyze the most important (psychological) driver behavior conceptualizations in order to identify crucial factors of human traffic behavior. Based on this analysis we examine the ability of state-of-the-art traffic simulation to account for these factors. It is our intention to determine the capabilities of traffic simulation frameworks, to point out perspectives for future research and to provide a guideline for the selection of the right traffic simulation system.
\end{abstract}

\section{INTRODUCTION}

Over the last decade, traffic simulation frameworks became an indispensible tool for traffic planning and infrastructure management. For these simulations, sophisticated models are used in order to mimic traffic systems in a lifelike fashion. In most cases, these models focus on a rather technical scope.

Human factors, such as the drivers' behavior are frequently neglected or focus on particular characteristics only. The reason for this is that human behavior "in its entirety" is difficult to understand, to capture and to formalize. Human behavior is highly individual and where a particular model fits for some characteristics, the same model may totally fail for others.

Nevertheless, there are some approaches that account for human factors. Most of these approaches use an agent-based model to formalize simulated drivers and traffic participants. Following Wooldridge and Jennings (1995) autonomous, re- and proactive, as well as socially acting entities can be considered as intelligent agents. This description almost perfectly fits for (simulated) traffic participants and for this reason, many software providers use the agent metaphor in order to make use of concepts, tools, development methodologies from the very established agent-domain.

Despite the comprehensive support, the conceptualization of behavioral aspects remains difficult-not least because only few works connect psychological findings with traffic simulation models. In this work we aim to tackle this problem and to establish such connection.

In order to do so, we first describe human driver behavior from a psychological point of view (see Section 2.1) and use this fundamental knowledge to analyze state-of-the-art works of human factor research in driver behavior (see Section 2.2). In this analysis we identify factors that determine human driver behavior. Later, in Section 3, we use these exact factors to determine the capability of contemporary traffic simulation frameworks to reproduce the distinct characteristics of human driving behavior. Finally, in Section 4 we conclude our work. 


\section{Lützenberger and Albayrak}

\section{DRIVING BEHAVIOR - A PSYCHOLOGICAL APPROACH}

In order to develop computable models for driving behavior it is necessary to understand the elements of human driving behavior as well as their assembly. Currently there are many traffic simulation frameworks that claim to realistically reproduce certain aspects of human driver behavior. Consider the ability to navigate or to account for different driving styles as examples for behavioral features. Nevertheless, there are only few works that use established models for the reproduction of driver behavior-even though there is an entire branch of research that is concerned with the development of such models, namely human factor psychology. We start this section by describing human driving behavior from a psychological point of view. In order to do so we present a work which significantly influenced our today understanding of driver behavior and proceed by presenting state-of-the-art driver conceptualizations. From this analysis we derive fundamental characteristics of human driving behavior. In the next section we use these findings to assess the capabilities of traffic simulation systems to reproduce human driving behavior.

\subsection{The Origin}

Our current understanding of human driving behavior begins with the work of John A. Michon. In his influential and widely cited article (Michon 1985), Michon presented an "informal" description of human driving behavior and used this description to analyze the capability of existing conceptualizations to "reproduce" his understanding of human driving behavior. As opposed to many earlier works, Michon (1985) stated that it is not possible to consider human mobility in isolation. Michon argued that human beings are always surrounded by a social as well as a technological environment and that any form of behavior can be considered as an interaction between the human being and such environment. Michon distinguished between four levels of interaction between the traffic system and human beings. Depending on the context, humans may either occur i) as an active road user ii) as a transportation consumer iii) as an active social being iv) or as a psycho-biological organism. The four levels of behavior are illustrated in Table 1.

Table 1: Behavioral levels relative to the hierarchical structure of problem solving tasks in traffic and transportation environments, adapted from Michon (1985).

\begin{tabular}{|c|c|c|c|c|}
\hline & \multicolumn{4}{|c|}{ Behavioral Level } \\
\hline & $\mathrm{I}$ & II & III & IV \\
\hline $\begin{array}{l}\text { Human Quality as } \\
\text { a Problem Solver }\end{array}$ & Road user & $\begin{array}{l}\text { Transportation } \\
\text { Consumer }\end{array}$ & Social Agent & $\begin{array}{l}\text { Psycho-biological } \\
\text { organism }\end{array}$ \\
\hline $\begin{array}{l}\text { Problem to be } \\
\text { solved }\end{array}$ & Vehicle Control & Trip making & $\begin{array}{l}\text { Activity pattern } \\
\text { (Communication) }\end{array}$ & $\begin{array}{l}\text { Satisfaction of ba- } \\
\text { sic needs }\end{array}$ \\
\hline Task environment & Road & $\begin{array}{l}\text { Road network (To- } \\
\text { pographical struc- } \\
\text { ture) }\end{array}$ & $\begin{array}{l}\text { Socio-economic } \\
\text { structure }\end{array}$ & $\begin{array}{l}\text { Nature } \\
\text { (environment) }\end{array}$ \\
\hline Task aids & $\begin{array}{l}\text { Vehicles, signs, } \\
\text { etc. }\end{array}$ & Transport mode & Transport system & $\begin{array}{l}\text { "Culture", } \\
\text { Technology }\end{array}$ \\
\hline
\end{tabular}

Michon (1985) used this informal description to assess the capabilities of existing behavior conceptualizations and concluded that examined approaches were not able to capture the dynamic interaction between human beings and their environment. Furthermore, Michon (1985) argued that existing conceptualizations focused on isolated parts of human driving behavior, a more "comprehensive" consideration was generally 


\section{Lützenberger and Albayrak}

neglected. Michon (1985) concluded that existing approaches consider behavior as a monolithic construct and do not account for the different roles in which humans occur (see above i-iv).

As a consequence, Michon proposed his own conceptualization. The Hierarchical Control Model (Michon 1985) explains driving behavior by means of different levels that are arranged in a loosely coupled hierarchy. In total, three behavioral levels were defined, namely the strategic level (planning), the tactical level (maneuvering) and the operational level (control):

The Strategic Level can be considered as the planning of an intended trip. At this stage, the trip goals are determined and a route is being calculated. Furthermore, the driver selects his means of travel and performs a cost-risk-analysis.

The Manoeuvring Level refers to tactical exercise maneuvers of the drivers. Actions that are performed on the maneuvering level, such as obstacle avoidance, lane change maneuvers, gap acceptance, turning, or overtaking have to be in compliance with the goals which have been set at the level above.

The Control Level refers to fundamental car controlling processes, such as controlling speed, following the road and keeping the vehicle on the road.

The different levels of behavior are arranged in a loosely coupled fashion, such that control may shift from one level to another in a timely manner, thus, the Hierarchical Control Model defines an interaction between the three levels of behavior and allows the outcome of lower levels to change goals and criteria that have been defined at a higher level and vice versa. The Hierarchical Control Model is illustrated in Figure 1.

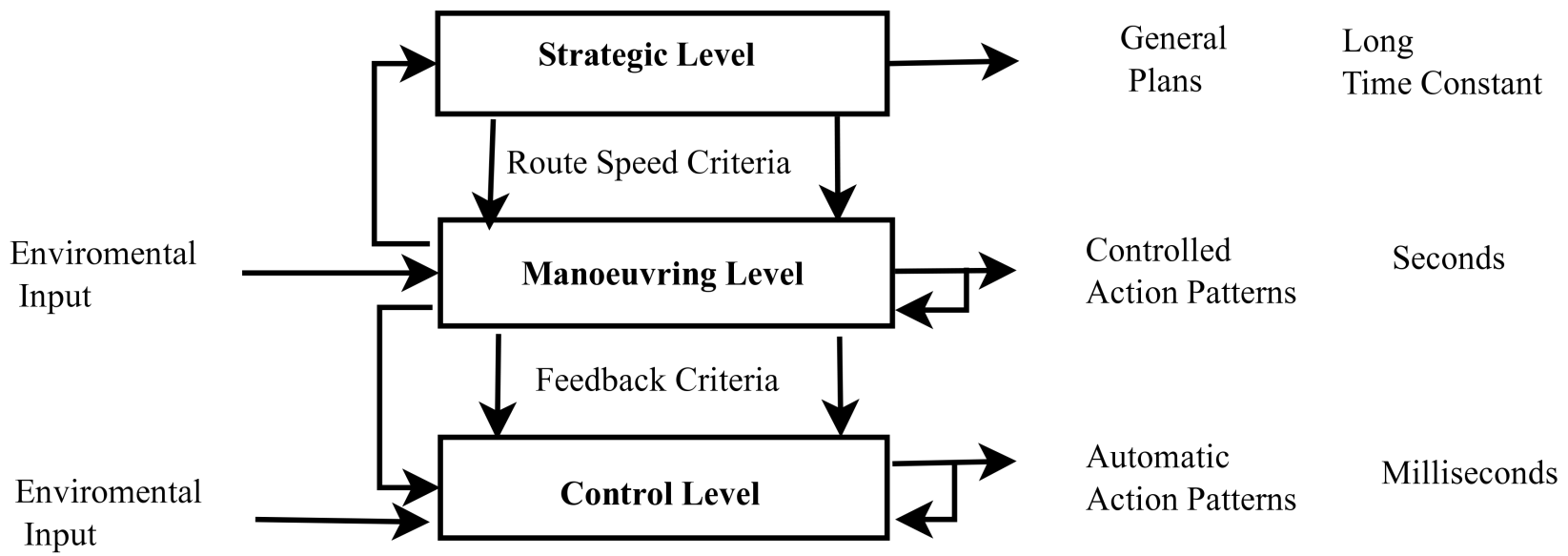

Figure 1: Michon's Hierarchical Control Model of the road user task. The three levels are loosely coupled. Internal and external outputs are illustrated as well (adapted from Michon 1985, Figure 2, p. 489).

The Hierarchical Control Model was the first approach that considered human driving behavior to comprise different levels. The model became generally accepted and helped the community of psychological driver research to gain new momentum. The Hierarchical Control Model was used as a foundation for many subsequent works and significantly shaped the most contemporary models of human driving behavior. In the following we provide an overview of state-of-the-art works in human driver behavior psychology.

\subsection{Driving Behavior - A State-of-the-Art}

The first approach we want to put a focus on is that of Keskinen et al. (2004), who extended Michon's hierarchy in a "vertical" fashion. Keskinen et al. (2004) defined an additional, a fourth level of driver behavior. At the lowermost level, the Vehicle Manoeuvring level, drivers account for the controlling of speed, the vehicle's direction and its position. This level has a strong resemblance to Michon's control level. One level above, at the Mastering Traffic Situations level, drivers adapt to the present traffic situation. 


\section{Lützenberger and Albayrak}

This level is similar to Michon's maneuvering level. On the next higher level, drivers deal with Goals and Context of Driving and consider their purpose, their environment their social context and their company. The level is similar to Michon's strategic level behavior, yet, the explicit consideration of the driver's environment was not described by Michon and can be considered as an extension in terms of strategic behavior. Finally, Keskinen defined an entirely new level at the top end of the behavioral hierarchy, namely the Goals for Life and Skills for Living level. This level covers the area of personality and motives and allows to describe behaviors which are "less congruent with the norms of the society" (Keskinen et al. 2004).

A similar approach was described by van der Molen and Bötticher (1988). The Hierarchical Risk Model for Traffic Participants (van der Molen and Bötticher 1988) describes human driving behavior in compliance with Michon's original hierarchy. In addition, the approach provides a concept for behavior alternatives and subjective probabilities and also accounts for perceptual, judgmental and decision processes of traffic participants at all levels of behavior. Furthermore, the model connects the drivers' strategic level behavior to their environment. The transitions between the different levels of behavior were refined as well. Information flow between the driver's strategic- and tactical level behavior was realized by so called Strategic Plans. Interaction between tactical- and the operational behavior was accomplished by so called Manoeuvring Plans.

As opposed to the first two approaches, Hale, Stoop, and Hommels (1990) extended Michons hierarchy not by further levels, but also by an additional dimension. On the vertical axis, the Matrix of Tasks (Hale, Stoop, and Hommels 1990) describes human driver behavior by means of Michon's original hierarchy. This hierarchy is extended by a "horizontal" dimension that accounts for the driver's expertise and his familiarity with the surrounding situation on each level of behavior. Furthermore, the model encompasses motivational aspects, which are able to affect a driver's behavior on each level of behavior. Hale, Stoop, and Hommels (1990) argue that the purpose and the importance of a trip may influence a driver throughout his journey. Yet, situations which are encountered "en route" may trigger short term goals that motivate tactical problem solving and lead to the same outcome. As an example, consider a driver who has selected a route and a departure time that ensures a leisurely and uneventful drive. The presence of extreme slow traffic ahead, may motivate even this driver to speed up and to pass. Furthermore, Hale, Stoop, and Hommels (1990) argue that strategic behavior is always a subject to the driver's experience, to his environment, and to his motivational aspects.

The next approach that we present is that of Summala (1996). The Filter Model of Risky Behaviour and Road Accidents (Summala 1996) conceptualizes human driving behavior by means of three dimensions. On the first dimension, Michon's original behavior hierarchy was used in order to account for vehicle choices, trip decisions, navigation, guidance and vehicle control. On the second dimension a functional taxonomy distinguishes between enclosed capabilities, such as lane keeping, headway control or obstacle avoidance, to name but a few. Finally, on the third dimension, three psychological processing levels are defined. The approach was extended to additionally account for motivational factors. Multiple Sieve Model (Summala 1997), explicitly accounts for emotions and motives and allows both factors to affect the drivers' behavior from strategic decisions to low-level vehicle control processes.

A very similar driver conceptualization was proposed by Hatakka et al. (1997). On the vertical axis, the GADGET-Matrix (Hatakka et al. 1997) uses Keskinen's levels of driver behavior (Keskinen et al. 2004). On the horizontal axis, high-order aspects of the driving task are arranged in three categories, namely knowledge and skills, risk-increasing factors and self-assessment. Knowledge and skills can be considered as routines and information that are required for driving under regular conditions, risk-increasing factors can be considered as traffic or life-related factors which are associated with a higher risk. Finally, selfassessment describes the drivers' ability to reflect their own driving skills and motivations. The hierarchical arrangement of the GADGET-Matrix indicates that decisions on the upper levels are able to affect the drivers' lower level behavior. Furthermore the GADGET-Matrix defines a connection between driver's attitudes, his lifestyle or his personal values and its strategic-level decisions. 


\section{Lützenberger and Albayrak}

Another relevant approach was proposed by Bekiaris, Amditis, and Panou (2003). The DRIVABILITY model (Bekiaris, Amditis, and Panou 2003) describes several factors that may simultaneously distract a driver's behavior. The fundamental assumption of the model is that driver behavior evolves over time as a result to five permanent and temporary contributors. i) individual resources are physical, social, psychological and mental conditions of the conceptualized driver, ii) knowledge and skills describe not only the driver's training and experience, but also knowledge in general. The reason for this is that basic education may influence a driver's motivation and his behavior. Furthermore, this contributor also comprises the driver's self-awareness for his own skills. iii) environmental factors describe the driver's environment. In addition to the status of his vehicle, this may also comprise traffic hazards, weather conditions, or the general traffic situation. Finally, iv\&v) workload and risk awareness are the two common denominators between the drivers' resources and their environmental status. The drivers' risk awareness is also influenced by other factors such as risk perception, the level of attention and potentially by driver support systems.

Salvucci, Boer, and Liu (2001) defined a similar model. The Adaptive Control of Thought-Rational, or ACT-R (Salvucci, Boer, and Liu 2001) combines cognitive, perceptual and motor dimension and was developed to encompass highway driving with moderate traffic. The ACT-R defines driver behavior by means of three different tasks, namely to control, to monitor and to decide. As opposed to Michon's suggestion to distinguish between different levels of behavior the ACT-R uses a monolithic representation of behavior. Yet, ACT-R emphasizes that a driver's decisions are subject to his personal attitudes as well as to external influences. Furthermore, ACT-R defines a connection between the driver's actions and their effects on the surrounding environment (Anderson et al. 2004).

The next relevant approach was presented by Krajzewicz and Wagner (2002). The A Common Mental Environment-Driver Model (or ACME-Driver Model) (Krajzewicz and Wagner 2002) is based on another, generally accepted, psychological model that was originally presented by Atkinson and Shiffring (1968). The ACME-Driver Model defines an architecture that is able to reproduce human decision-making processes in traffic situations. The proposed architecture comprises three different types of memories, namely the sensoric input register, the short-term memory and the long-term memory. The main difference between the memory types is the time collected information remain available. The model complies with the work of Tulving (1972) and thus subdivides the long-term memory into i) an episodic memory, which saves information about single situations from the human's life ii) the semantic memory, which is used to save common or logically expressible rules such as rules of algebra, and iii) the procedural memory, which contains non-verbalisable information about movements. The ACME-Driver model does not account for behavior levels and the authors explicitly argue that the ACME-Driver model was not supposed to serve as a comprehensive driver conceptualization, but rather as a detailed description of a driver's operational level of behavior. The ACME-Driver Model emphasizes the importance of external factors and long-term aspects such as experiences and strategies for the outcome of cognitive processes that evolve from the drivers' short term memory module.

The last approach that we want to present is the Contextual Control Model, or COCOM (Hollnagel 1993). The COCOM is based on the perceptual cycle (Neisser 1976) and considers the controller as well as the controlled system as a joint cognitive system rather than focusing on both entities in isolation. The central concept of the COCOM model is the so called construct-action-event cycle, which describes how a controller selects his actions based on his knowledge or his assumptions about the situation in which the action takes place. The construct-action-event cycle further provides a concept to describe the consequences of the selected actions on the controlled system. In short, the selected actions generate new events that are again perceived by the controller.

The one-dimensional structure of the COCOM was extended to additionally account for different levels of driver behavior. Its successor, the Extended Control Model (ECOM) (Hollnagel and Woods 2005, Engström and Hollnagel 2007) combines the COCOM and Michon's hierarchy and thus conceptualizes cognitive control processes on different levels of behavior. Engström and Hollnagel (2007) describe human traffic behavior by means of four behavioral levels, namely tracking, regulating, monitoring and targeting. 


\section{Lützenberger and Albayrak}

On the targeting level the driver sets general goals of the driving task. These goals constitute the input for the next level (the monitoring level) on which the driver attempts to control the state of the joint vehicledriver system relative to the driver's environment. Among others, the monitoring level comprises tasks like monitoring properties of the traffic environment (such as speed limits) or the location and the condition of the vehicle. On the regulating level, the driver deals with conscious processes, such as keeping desired safety margins to other traffic elements. Finally, on the tracking level, the driver performs momentary and automated corrections to external disturbances, such as wind gusts.

To sum up-above we have presented selected state-of-the-art solutions for the psychological conceptualizations of driver behavior. In the following, we analyze the presented approaches and determine similarities as well as factors that determine human traffic behavior. Later we use these findings to assess the capabilities of existing traffic simulation frameworks.

\subsection{Implications}

Although the presented approaches were developed independently from each other, the capabilities of the models surprisingly converge.

To start with-most presented models (cf. Engström and Hollnagel 2007, Hale, Stoop, and Hommels 1990, Hatakka et al. 1997, Keskinen et al. 2004, Summala 1996, Summala 1997, van der Molen and Bötticher 1988) understand human driver behavior as a hierarchically ordered control structure where several levels of behavior interact in a flowing transition and respectively allow the outcome of lower levels to change goals and criteria that have been defined at a higher level. This structure was originally proposed by Michon (1985) and was refined ever since. We conclude that driver behavior comprises different levels, and where the lower levels deal with the driving in a technical sense (e.g. shifting gears, steering), the upper levels account for aspects that are not entirely related to the driving itself (e.g. navigating, route choices, or to select means of transportation). Some approaches (e.g. Keskinen et al. 2004, or Engström and Hollnagel 2007) go further and account for meta aspects, such as the drivers' goals for life.

The second trend that can be observed is that a driver's behavior is connected to its environment (cf. Bekiaris, Amditis, and Panou 2003, Engström and Hollnagel 2007, Hale, Stoop, and Hommels 1990, Hollnagel 1993, Keskinen et al. 2004, Krajzewicz and Wagner 2002, Salvucci 2006, van der Molen and Bötticher 1988). It is important to mention that effects of external factors comprise the entire behavioral hierarchy of the driver and not only the lower levels, where such dependency is somehow obvious. As an example for environmental affection of a driver's maneuvering-behavior consider the sudden breaking of a preceding vehicle, which causes a pursuer to adapt his velocity in order to avoid an accident. However, the analysis clearly demonstrated that external factors also affect the upper levels of behavior. As an example for this connection consider a driver with a high affinity for public transport that might change his means of transportation when confronted with extreme weather conditions (e.g. freezing rain, or heavy snowfalls) near a metro station and available parking (Lützenberger et al. 2012). As another example, consider the occurrence of congestion that may cause isolated drivers to avoid critical road sections and to deviate from their originally selected strategy (Lützenberger et al. 2012). Based on our analysis we conclude that any simulation model that is used to generate strategic-level behavior of drivers has to define a connection between the module that generates strategic-level behavior and the driver's environment. Most existing simulation frameworks define such connection for the lower levels of behavior, only.

Thirdly, our analysis substantiated the importance of the driver's emotional state and its motives. Both factors evolve from the driver, and therefore we refer to both as "internal factors". Most examined approaches (cf. Bekiaris, Amditis, and Panou 2003, Hale, Stoop, and Hommels 1990, Hatakka et al. 1997, Salvucci 2006, Summala 1997, van der Molen and Bötticher 1988) account for internal factors and define a dependency between the drivers' mental state and their behavior. Again, this dependency comprises the entire behavioral hierarchy, such that, emotions may affect a driver's route selection, breaking or lane-changing behavior and technical procedures such as shifting the gear. 


\section{Lützenberger and Albayrak}

The last aspect to mention is the concept of familiarity and automatisms. In chronological terms, Hale, Stoop, and Hommels (1990) were the first to expand Michon's vertical arrangement by horizontal factors and thus described human driver behavior by means of more than one dimension. On the vertical axis, Hale, Stoop, and Hommels (1990) used Michon's hierarchy, though, in horizontal terms, differing levels of automatisms and "familiarity" were added. Based on our survey we conclude that such concept is a key factor for human traffic behavior. Most examined approaches (cf. Hatakka et al. 1997, Krajzewicz and Wagner 2002, Summala 1996, Summala 1997) account for the concepts of familiarity and automatisms.

Based on our analysis of psychological works we have identified four factors that determine human driver behavior. These factors include i) different levels of behavior, which are vertically assembled and affect each other, ii) environmental factors, which affect each level of behavior iii) emotion and motives, or internal factors, which evolve from the drivers and affect their behavior on each level, iv) aspects of familiarity and automatisms, which account for different levels of expertise and skills.

In the following we analyze contemporary traffic simulation frameworks and simulation models for human traffic behavior in order determine the capabilities of theses frameworks to reproduce human driving behavior.

\section{TRAFFIC SIMULATION SYSTEMS AND MODELS}

Over the last decade, traffic simulation frameworks have become an indispensible tool for traffic planning and infrastructure management. These frameworks use sophisticated models to reproduce traffic systems in a lifelike fashion. Most of the applied models, however, focus on technical aspects and neglect human factors, such as the drivers' behavior. In fact, there are some approaches that use conceptualizations of driver behavior for traffic simulations (cf. Ehlert and Rothkrantz 2001, Krajzewicz 2010, Fellendorf and Vortisch 2010, Sykes 2010, Beuck et al. 2008). In most cases, an agent-based model is used as a foundation. The reason for the application of agent-based techniques is the nature of the multi-agent system paradigm, which considers i) autonomous, ii) reactive, iii) proactive, and iv) socially competent program parts as software agent (Wooldridge and Jennings 1995) - this system description, almost naturally fits for simulated traffic participants. In this section we analyze contemporary traffic simulation frameworks and simulation models and identify the capability of these solutions to reproduce the essential characteristics of human driving behavior, which we compiled in the previous section. We start by describing approaches that account for more than one behavioral feature and present approaches that account exactly one feature, afterwards.

\subsection{Multi Behavior Frameworks}

The first approach to mention is the Simulation of Urban Mobility (or SUMO) framework (Krajzewicz 2010). SUMO is an open-source, microscopic traffic simulation package that has been developed as a test-bed for research matters. The software is able to handle large road networks and can be extended easily. SUMO simulates driver behavior in compliance with the ACME-Driver Model (Krajzewicz and Wagner 2002). The model is able to produce elementary forms of strategic-level behavior, such as individual routing capability or the ability of drivers to recognize high congestion and use alternative routes. Maneuvering level behavior is realized by advanced longitudinal (Krauß 1998) and lateral models (Krajzewicz 2009). Simulated drivers recognize congestion, therefore the framework defines a connection between the simulated drivers and their environment. Nevertheless, a more comprehensive perception is not realized. As an example, simulated drivers are not able to perceive weather conditions or the infrastructure which surrounds them. SUMO does not allow to include such factors in the simulation topology and provides no concept for drivers to perceive their surrounding infrastructure. Nevertheless, it should be mentioned that the SUMO framework is one of the most advanced applications that is currently available.

The second framework that falls under the umbrella of multi-behavior frameworks is MATSim (Beuck et al. 2008). MATSim supports large-scale agent-based transport simulations and consists of several modules which can be use either in combination or isolated. For the conceptualization of the driver 


\section{Lützenberger and Albayrak}

behavior, MATSim uses an agent-based approach (Balmer et al. 2004). The simulated world comprises i) a physical layer, where agents move and avoid each other, and ii) a mental layer, where agents generate strategies such as routes, mode choices and daily activity plans. Based on the applied agent model, MATSim is able to simulate elementary forms of strategic-level behavior. Similar to the capabilities of the SUMO framework, MATSim provides a concept for individual route choices and the avoidance of congested road sections. Furthermore, mode choices are supported. This implies that the simulated agents perceive their environment and are aware of transport options (e.g. public transport). Nevertheless, MATSim provides no generic model for external factors that evolve from the environment and neglects emotions, motivations and the experience of the drivers entirely.

\subsection{Environmental Factors}

The VISSIM Framework (Fellendorf and Vortisch 2010) is a commercial simulation software for multi-modal traffic flow modeling. With a high level of detail VISSIM accurately simulates urban and highway traffic, including pedestrians, cyclists and motorized vehicles. VISSIM is able to simulate the parking search process and thus includes information about the environment which can be processed by the simulated drivers. A similar feature is provided by Paramics (Sykes 2010). Other approaches, such as MITSIMLab (Ben-Akiva et al. 2010) or Aimsun (Casas et al. 2010) also define an interaction between the simulated drivers and their environment. Both approaches include information on public transportation stops into their simulation topology and use a driver model which is able to perceive such information. Finally, there are approaches which allow for the specification of particular weather conditions such as heavy rain, ice or snow. One particular approach that is able simulate different weather conditions is AVENUE (Kuwahara, Horiguchi, and Hanabusa 2010). Similar models were described by Kyte et al. (2001) and Rigolli and Brady (2005).

\subsection{Attitudes and Emotions}

There are many approaches that account for attitudes and emotions of the simulated drivers. The most popular framework was developed by the French automobile manufacturer Renault. The SCANeR II simulator (Champion et al. 1999) was used for ergonomics and advanced engineering studies, for research in road traffic, for human factor studies, and for driver training. SCANeR II is an agent-based traffic simulator and features microscopic simulation of interactively driven entities. Currently, vehicles, trucks, motorcycles, bikes, train, trams or pedestrians are supported. Simulated objects are able to either mimic "unique" or "risky" driving behavior. Another approach was presented by Paruchuri, Pullalarevu, and Karlapalem (2002). The authors describe a microscopic simulation framework that considers vehicles as autonomous software agents. The authors use micro- and macro goals for the realization of different driving styles, such as "aggressive", "normal" and "cautious". Furthermore psychological traits, such as "confidence" and "rush" are implemented as well. Tang and Wan (2005) describe a multi-agent traffic simulation system for urban environments, which is capable of modeling traffic in response to current road network loading at the time of interest. Ehlert and Rothkrantz (2001) describe a microscopic traffic simulator and a model for reactive software agents which are able to control the simulated vehicles. The simulator supports simulations within an urban environment with multi-lane roads, intersections, traffic lights and traffic lights controllers. The described model works fine and is able to realize individual driving styles from slow and careful to fast and aggressive.

\subsection{Levels of Automatism and Experience}

We are not aware of any traffic simulation systems or computable driver models which account for the human concept of automatism or includes a concept to define experiences of drivers. 


\section{Lützenberger and Albayrak}

\subsection{Implications}

Above we have presented the most popular frameworks and approaches that account for the simulation-based reproduction of human driving behavior.

The most advanced frameworks (in terms of behavior aspects) are without a doubt SUMO (Krajzewicz 2010) and MATSim (Beuck et al. 2008). In both frameworks, simulated drivers autonomously determine routes, determine strategies to avoid congested road sections and-in the case of MATSim-decide on when to use public transportation instead of vehicles. Nevertheless, in the light of our analysis from the previous section, we want to mention that both frameworks fall short in accounting for high-level behavior. As an example, it is not possible to define a driver's goals for life and thus to account for particular driving characteristics that evolve from this behavioral level. As for the strategic level behavior both frameworks produce, we have to note that a general consideration is generally missing. Both frameworks focus on route finding abilities and reactions to congestion. There is no dynamic interaction between the drivers' infrastructure and their behavior. Finally, there is no concept for emotions, attitudes or different levels of automatism and experience. Both frameworks feature a high level of sophistication, nevertheless, we were able to define a certain discrepancy between the capabilities of psychological conceptualizations and the kind of behavior simulation based approaches are able to produce. We explain this discrepancy with the area of application both frameworks were designed for. Both frameworks were originally developed to analyze the effects of microscopic maneuvers on large road networks. Thus, in order to microscopically simulate thousands of vehicles, the developers used very quick longitudinal and lateral models and geared the drivers' behavior capabilities towards the purpose of the frameworks. As such, both frameworks provide fairly extensive implementations of the controlling- and maneuvering levels but fall short in the strategic level behavior they produce. SUMO equips drivers with route finding abilities and allows them to recognize congestion. MATSim provides similar features and additionally allows drivers to recognize (parts) of their infrastructure, that is, bus- and metro stops and to consider these transport options for their route finding capability. Higher levels of behavior, attitudes and emotions and aspects of automatism and experience are neglected from both frameworks. Nevertheless, whenever there are questions that require the consideration of the lower levels of driving behavior (the maneuvering- and the control level) or elementary strategic abilities (route finding and reactions on high congestion), one is advised to use either MATSim or the SUMO framework.

Other approaches focus on isolated characteristic of human driver behavior. We were able to identify simulation frameworks (cf. Ben-Akiva et al. 2010, Casas et al. 2010, Fellendorf and Vortisch 2010, Kuwahara, Horiguchi, and Hanabusa 2010, Kyte et al. 2001, Rigolli and Brady 2005, Sykes 2010) where the driver's behavior is somehow connected to their environment. Most frameworks focus on specific external factors, such as parking lots, bus- or metro stops, or extreme weather conditions. A more general consideration, however, is not done. There are particular cases, where a more general consideration is useful-especially when the interdependency between two- or more external factors has to be examined), nevertheless, when one wants to examine the effects of external conditions on road networks, one is advised to use on of the above-mentioned frameworks.

We also identified many works (cf. Champion et al. 1999, Ehlert and Rothkrantz 2001, Paruchuri, Pullalarevu, and Karlapalem 2002, Tang and Wan 2005), with a focus on the representation of attitudes and emotions of the simulated drivers. The approaches produce reliable results and are used to examine the effects of driving styles on certain traffic situations. Whenever the effects of internal factors on road networks has to be analyzed, the above-mentioned works can be used.

Finally, we can say that we were not able to identify any concepts of automatism or experience which can be used for traffic simulation frameworks. It is our opinion that such concepts are important in order to simulate and to understand traffic. The ability "to learn" and to "collect information and experience" are vital factors for a reliable representation of traffic behavior. 


\section{Lützenberger and Albayrak}

\section{CONCLUSION}

Traffic simulation frameworks have reached a high degree of realism and sophistication. Despite the rich offer, it is our opinion, that behavioral aspects of the simulated drivers are not comprehensively covered. In order to substantiate this thesis, we analyzed psychological models and driver conceptualizations and derived structural dependencies and particular features and characteristics of human driving behavior. Following the most popular psychological approaches, human driving behavior: i) comprises different, hierarchically ordered levels, ii) is connected to the drivers' environment, iii) is influenced by internal attributes, such as attitudes and emotions and iv) is determined by the drivers' level of expertise. After we have determined the characteristics of human driving behavior, we analyzed contemporary traffic simulation frameworks and assessed the frameworks' capabilities to reproduce human driving behavior. From the analyzed works, we were not able to determine a framework which is able to completely comprehend the psychological understanding of human driving behavior. We identified two frameworks, namely SUMO (Krajzewicz et al. 2002) and MATSim (Beuck et al. 2008) that accounted for more than one characteristic of human driving behavior. Both frameworks account for the hierarchical arrangement of different behavior levels and provide fairly comprehensive implementations for the lower layers. Furthermore, individual elements of high-level driving behavior, such as route finding and congestion avoidance are realized. Beyond that, the MATSim framework defines a connection between the drivers' behavior and their infrastructure. Simulated drivers are able to perceive nearby bus- and metro stations and to consider their perception for route decisions. We advise to make use of these frameworks whenever there are questions that require to consider the lower levels of driving behavior (the maneuvering- and the control level) or elementary strategic abilities (route finding and reactions on high congestion). In addition to SUMO and MATSim, we identified several frameworks with the ability to reproduce isolated characteristics of human driving behavior. There were many approaches (cf. Ben-Akiva et al. 2010, Casas et al. 2010, Fellendorf and Vortisch 2010, Kuwahara, Horiguchi, and Hanabusa 2010, Kyte et al. 2001, Rigolli and Brady 2005, Sykes 2010), which account for a connection between the drivers' behavior and their environment. Most approaches focus on particular aspects, such as the effects of new car parks, public transportation or extreme weather situations on the drivers' behavior and the traffic system respectively. A more general model is not provided. Nevertheless, when one wants to examine the effects of external conditions on road networks, one is advised to use on of the above-mentioned frameworks. There were also many frameworks (cf. Champion et al. 1999, Ehlert and Rothkrantz 2001, Paruchuri, Pullalarevu, and Karlapalem 2002, Tang and Wan 2005) that account for attitudes and emotions of simulated drivers. The approaches produce reliable results and are frequently used to investigate the effects of driving styles on certain traffic situations. Whenever the effects of internal factors on road networks have to be analyzed, the above-mentioned works can be used. Finally we were not able to determine any frameworks, which account for concepts of automatism or experience of the simulated drivers. It is our opinion that such concepts are important in order to simulate and to understand traffic. The ability "to learn" and to "collect information and experience" are vital factors for a reliable representation of traffic behavior. Our analysis showed that there are many traffic simulation frameworks, which do account for the driver's behavior. Nevertheless, the capabilities of these frameworks do not entirely match psychological models. While most frameworks consider isolated features of human driving behavior, a more comprehensive connection is not done. In order to reproduce human driving behavior in its entirety, such connection should be a subject to future research.

\section{REFERENCES}

Anderson, J. R., D. Bothell, M. D. Byrne, S. Douglass, C. Lebiere, and Y. Qin. 2004. "An Integrated Theory of the Mind". Psychological Review 111:1036-1060.

Atkinson, R. C., and R. M. Shiffring. 1968. "Human Memory: A Proposed System and its Control Processes". In The Psychology of Learning and Motivation: Advances in Research and Theory, edited by K. W. Spence, 89-195. Academic Press, Inc. 


\section{Lützenberger and Albayrak}

Balmer, M., N. Cetin, K. Nagel, and B. Raney. 2004. "Towards Truly Agent-Based Traffic and Mobility Simulations". In Proceedings of the $3^{\text {rd }}$ International Joint Conference on Autonomous Agents and Multiagent Systems, AAMAS '04, 60-67. Washington, DC, USA: IEEE Computer Society.

Barceló, J. (Ed.) 2010, October. Fundamentals of Traffic Simulation. $1^{\text {st }}$ ed, Volume 145 of International Series in Operations Research and Management Science. Springer.

Bekiaris, E., A. Amditis, and M. Panou. 2003. "DRIVABILITY: a new concept for modelling driving performance". Cognition, Technology \& Work 5:152-161.

Ben-Akiva, M., H. N. Koutsopoulos, T. Toledo, Q. Yang, C. F. Choudhury, C. Antoniou, and R. Balakrishna. 2010, October. "Traffic Simulation with MITSIMLab”. See Barceló (2010), 233-268.

Beuck, U., K. Nagel, M. Rieser, D. Strippgen, and M. Balmer. 2008. "Preliminary Results of a Multi-Agent Traffic Simulation for Berlin". In The Dynamics of Complex Urban Systems, edited by S. Albeverio, D. Andrey, P. Giordano, and A. Vancheri, 75-94. Physica-Verlag HD.

Casas, J., J. L. Ferrer, D. García, J. Perarnau, and A. Torday. 2010, October. "Traffic Simulation with Aimsun”. See Barceló (2010), 173-232.

Champion, A., R. Mandiau, C. Kolski, A. Heidet, and A. Kemeny. 1999. "Traffic generation with the SCANeR ${ }^{\circledR}$ II simulator: towards a multi-agent architecture”. In Proceedings of the $1^{\text {st }}$ Driving Simulation Conference, Paris, France, 311-324.

Ehlert, P. A. M., and L. J. M. Rothkrantz. 2001. "A Reactive Driving Agent for Microscopic Traffic Simulations". In Proceedings of the $15^{\text {th }}$ European Simulation Multiconference, Prague, Czech Republic, 943-949: SCS Publishing House.

Engström, J., and E. Hollnagel. 2007. "A General Conceptual Framework for Modelling Behavioural Effects of Driver Support Functions". In Modelling Driver Behaviour in Automotive Environments ( $1^{\text {st }}$ ed.)., edited by C. Cacciabue, 61-84. Springer.

Fellendorf, M., and P. Vortisch. 2010, October. "Microscopic Traffic Flow Simulator VISSIM". See Barceló (2010), 63-94.

Hale, A. R., J. Stoop, and J. Hommels. 1990. "Human Error Models as Predictors of Accident Scenarios for Designers in Road Transport Systems". Ergonomics 33 (10-11): 1377-1387.

Hatakka, M., E. Keskinen, E. Katila, and S. Laapotti. 1997. "Do psychologists have something to offer in driver training, driver improvement and selection?". In Assessing the driver, edited by R. Risser, Number 41 in Faktor Mensch im Verkehr.

Hollnagel, E. 1993. Human Reliability Analysis: Context and Control. Computers and Peoples Series. Academic Press, Inc.

Hollnagel, E., and D. D. Woods. 2005. Joint Cognitive Systems - Foundations of Cognitive Systems Engineering. CRC Press.

Keskinen, E., M. Hatakka, S. Laapotti, A. Katila, and M. Peräaho. 2004. "Driver Behaviour as a Hierarchical System". In Traffic \& Transport Psychology: Theory and Application $\left(1^{\text {st }}\right.$ ed.)., edited by T. Rothengatter and R. D. Huguenin, 9-23. Elsevier.

Krajzewicz, D. 2009. "Kombination von taktischen und strategischen Einflüssen in einermikroskopischen Verkehrsflusssimulation". Volume 28 of Fortschritt-Berichte, 104-115. VDI-Verlag.

Krajzewicz, D. 2010, October. "Traffic Simulation with SUMO - Simulation of Urban Mobility". See Barceló (2010), 269-294.

Krajzewicz, D., G. Hertkorn, P. Wagner, and C. Rössel. 2002, September. "SUMO (Simulation of Urban MObility) - An open-source traffic simulation". In Proceedings of the $4^{\text {th }}$ Middle East Symposium on Simulation and Modelling, Sharjah, United Arab Emirates, 183-187.

Krajzewicz, D., and P. Wagner. 2002. "ACME (A Common Mental Environment)- Driver A Cognitive Car Driver Model". In Proceedings of the 16th European Simulation Multiconference on Modelling and Simulation, 689-693: SCS Europe.

Krauß, S. 1998. Microscopic Modeling of Traffic Flow: Investigation of Collision Free Vehicle Dynamics. $\mathrm{Ph}$. D. thesis. 


\section{Lützenberger and Albayrak}

Kuwahara, M., R. Horiguchi, and H. Hanabusa. 2010, October. "Traffic Simulation with AVENUE". See Barceló (2010), 95-130.

Kyte, M., Z. Khatib, P. Shannon, and F. Kitchener. 2001. "Effect of Weather on Free-Flow Speed". Transportation Research Record: Journal of the Transportation Research Board 1776:60-68.

Lützenberger, M., S. Ahrndt, B. Hirsch, N. Masuch, A. Heßler, and S. Albayrak. 2012. "Reconsider Your Strategy - An Agent-Based Conceptualisation of Compensatory Driver Behaviour". In Proceedings of the $15^{\text {th }}$ Intelligent Transportation Systems Conference, Anchorage, AK, USA, 340-346.

Michon, J. A. 1985. "A Critical View of Driver Behaviour Models: What do we know, what should we do?". In Human Behavior and Traffic Safety, edited by L. Evans and R. C. Schwing, 487-525. Plenum Press.

Neisser, U. 1976. Cognition and Reality - Principles and Implications of Cognitive Psychology. W. H. Freeman and Company.

Paruchuri, P., A. R. Pullalarevu, and K. Karlapalem. 2002. "Multi Agent Simulation of Unorganized Traffic". In Proceedings of the $1^{\text {st }}$ International Joint Conference on Autonomous Agents and Multiagent Systems, Bologna, Italy, 176-183.

Rigolli, M., and M. Brady. 2005. "Towards a Behavioural Traffic Monitoring System". In Proceedings of the $4^{\text {th }}$ International Joint Conference on Autonomous Agents and Multiagent Systems, Utrecht, The Netherlands, 449-454: ACM.

Salvucci, D. D. 2006. "Modeling Driver Behavior in a Cognitive Architecture". Human Factors 48 (2): 362-380.

Salvucci, D. D., E. R. Boer, and A. Liu. 2001. "Toward an integrated model of driver behavior in a cognitive architecture". Transportation Research Record 1779:9-16.

Summala, H. 1996. "Accident risk and driver behaviour". Safety Science 22 (1-3): 103-117.

Summala, H. 1997. "Hierarchical Model of Behavioural Adaptation and Traffic Accidents". In Traffic \& Transport Psychology: Theory and Application, edited by T. Rothengatter and E. Vaya, 41-52. Pergamon, Elsevier Science, U.K.

Sykes, P. 2010, October. "Traffic Simulation with Paramics”. See Barceló (2010), 131-172.

Tang, W., and T. R. Wan. 2005. "Multi-agent Animation Techniques for Traffic Simulation in Urban Environment". In Proceedings of the $13^{\text {th }}$ Winter School of Computer Graphics, Plzeñ, Czech Republic, 161-164.

Tulving, E. 1972. "Episodic and Semantic Memory". In Organization of Memory, edited by E. Tulving and W. Donaldson, Chapter Episodic and Semantic Memory, 381-403. Academic Press, Inc.

van der Molen, H. H., and A. M. T. Bötticher. 1988. "A hierarchical risk model for traffic participants". Ergonomics 31 (4): 537-555.

Wooldridge, M., and N. R. Jennings. 1995, June. "Intelligent Agents: Theory and Practice". Knowledge Engineering Review 10 (2): 115-152.

\section{AUTHOR BIOGRAPHIES}

MARCO LÜTZENBERGER received a diploma in computer science in 2009 from the Technische Universität Berlin. From 2009 until now, he is part of the Competence Center Agent Core Technologies at the DAI-Lab, where he is working on his doctoral dissertation in the field of multi-agent based simulation models. His email address is marco.luetzenberger@dai-labor.de.

SAHIN ALBAYRAK is Professor of Agent Technologies in Business Applications and Telecommunication at Technische Universität Berlin. He is the founder and head of the DAI-Lab, currently employing about one hundred researchers and support staff. His email address is sahin.albayrak@dai-labor.de. 